\title{
Interference Management Techniques in Small Cells Overlaid Heterogeneous Cellular Networks
}

\author{
Soumya Ranjan Samal \\ Faculty of Telecommunications, Technical University of Sofia, Bulgaria \\ E-mail: sranjansamal@tu-sofia.bg \\ Received 10 January 2018; Accepted 27 April 2018; \\ Publication 3 July 2018
}

\begin{abstract}
Now-a-days because of the rapid increase in mobile users mobile data has also raised. Due to the heavy demand of data rates for advanced applications service providers are forced to adopt different technological advancements in conventional networks. Looking toward this context heterogeneous networks (HetNets) play a vital role in future 5G wireless cellular network deployment. In Heterogeneous Cellular Networks (HCNs) low power small cells are overlaid with the existing macro-only cell which increases the complexity of the network. Some of the emerging network technologies for this cellular evolution are the Femtocell, Picocell and Metrocell networks. Due to the deployment of these small cells in HCNs there are wide varieties of network structures and different transmitting powers which lead to interference in the cellular network. In addition to this, due to the spatiotemporal distribution of the mobile users and their mobility, hotspots also lead to increase of the possibilities of interference generation in wireless cellular network systems. The major technical issues associated with the mass deployment of smaller cells are related to the interference management between smallcells i.e. Femtocells and other serving cells in the same spectrum. Since Femtocells and Macrocells share the same frequency bands, it is necessary to develop efficient interference management techniques to increase the capacity and throughput of HCNs. So in order to meet the user demands in an efficient way
\end{abstract}

Journal of Mobile Multimedia, Vol. 14_3, 273-306.

doi: 10.13052/jmm1550-4646.1432

(c) 2018 River Publishers 
without affecting the quality of service (QoS) and throughput of the network, many interference management schemes and power control approaches have been proposed for successful deployment of HCNs. In this paper a review with a brief analysis of some of the efficient interference management techniques along with their challenges is proposed, while keeping the main focus on the hardware interference management techniques. An overview on the coverage and capacity optimization problems in a multi-tier cellular network is also presented.

Keywords: Interference, Interference management, Heterogeneous Cellular Network, Small Cells, Cell Less, Closed Subscriber Group.

\section{Introduction}

Due to extensive use of data hungry devices (smart-phones, tablets etc.), for content rich multimedia applications, mobile data traffic has increased many fold in the last two decades and the same trend is forecasted to be continued [1]. It is being estimated by Cisco that the global mobile data traffic has grown up to 11.2 Exabyte per month in 2017 [2] and according to Ericson the mobile broad band users will be multiplied [3]. In this context, it is assumed that the future wireless communication technologies are expected to attain 10-100 times higher number of connecting devices and user data rate, 1000 times higher mobile data volume per unit area, 5 times reduced latency and 10 times longer battery life [4]. Hence, disruptive changes and challenges for the telecommunication industry are multidirectional: increase in times of the data capacity of the network within pre-allocated spectrum without affecting the required QoS while reducing the cost per bit [5].

The classical approach to handle ever-increasing traffic is network densification through cell splitting. The bits/sec/ $\mathrm{Km}^{2}$ metric of the network is improved by replacing a big cell into number of smaller cells of the same class; each small cell consisting of a single transmitter. With its continuous reduction in the last two decades the cell size has been reduced to fraction of square kilometers. Hence, a recent approach implemented for network densification is the HetNet, where more than one class of transmission nodes are being deployed in same area. In a HetNet, several lower power tiers like Picocell, Femtocell and relay co-exists along with long range coverage by traditional macro base stations. The schematic representation of a HetNet is shown in Figure 1. 


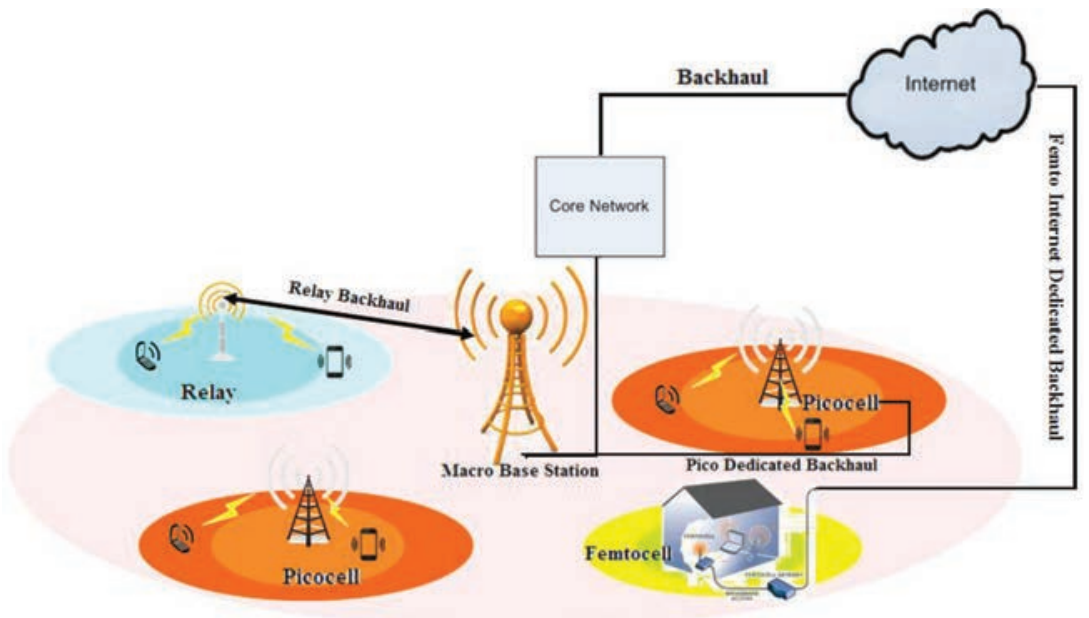

Figure 1 Schematic representation of HetNet.

In general a HetNet consists of the following various elements [6]:

- A Macrocell is the conventional operator-installed base station (BS) which provides an open access to the public with coverage of about a few kilometers. In LTE, they are also called enhanced NodeBs (eNBs). They use a dedicated backhaul and are able to provide a guaranteed minimum data rate under maximum tolerable delay and outage constraints to more than thousands of customers. These Macrocells typically have a transmit power of up to $46 \mathrm{dBm}$.

- Low-power operator-installed Picocells which use the same backhaul and access features as Macrocells. They have the capability to provide a coverage area of $300 \mathrm{~m}$ (usually between 40-75 m [7] or less) and are used to serve few numbers of users. Picocells are used to increase the capacity as well as the indoor and outdoor coverage with a transmit power about $30 \mathrm{dBm}$.

- Femtocells are considered as low-power user deployed access points which use consumers' broadband connections (DSL) for data traffic offloading. These cells are also known as home BSs (HeNBs) and basically used to serve a few active users in homes or enterprises in an Open or Closed Subscriber Group (CSG).The area of coverage of a Femtocell is about less than $50 \mathrm{~m}$ with a transmitting power of less than $23 \mathrm{dBm}$. 
- Relays are usually operator-deployed access points used to improve the signal strength in poor coverage area i.e. inside tunnels, cell edges etc., depending upon their position in the existing networks. They can operate in transparent or non-transparent modes (e.g., IEEE 802.16m).

- Radio Relay Heads (RRHs) are high-power, compact-size, and lowweight units connected to a conventional macro BS with the help of a fiber optic cable creating a distributed BS. The RRHs are generally mounted outside the conventional BS where the central macro BS is in charge of control and baseband signal processing. The physical limitations and site acquisition for network deployment can be reduced by RRHs.

A comparison of various elements used in HetNet is given in Table 1:

The implementation of HetNets can lead to a significant improvement in the areal spectral efficiency (bits/sec/ $\mathrm{Km}^{2}$ ) through extreme reuse of spectrum across a given geographic area. This is due to the fact that, the user examines the strengths of the received signals and chooses a single node from any tier as its servicing node depending on the user association algorithm used. This process results significant offload of traffic to lower tiers from macro-tier to achieve maximum throughput. However, in this type network topology, the user treats one signal as its intended signal but the rest of the signals as interference. Hence, the major drawback in successful commercial implementation of a HetNet is the low signal-to-interference-plus-noise-ratio (SINR). Various QoS parameters of the network like throughput, bit error rate (BER) and outage probability are directly related to SINR. Hence, major focus of this overview is related to the interference and related interference management approaches in HetNets. In order to fulfill the increased traffic demand, another aspect of

Table 1 Comparison of various elements of HetNet

\begin{tabular}{|c|c|c|c|c|c|c|}
\hline Node Type & $\begin{array}{l}\text { Tx Power } \\
(\mathrm{dBm})\end{array}$ & $\begin{array}{l}\text { Area of } \\
\text { Coverage }\end{array}$ & Backhaul & Deployment & $\begin{array}{l}\text { Modes of } \\
\text { Access }\end{array}$ & CAPEX \\
\hline Microcell & 46 & Few KM & S1 interface & Outdoor & Open to all & Expensive \\
\hline Picocell & $23-30$ & $<300 \mathrm{~m}$ & $\begin{array}{l}\mathrm{X} 2 \\
\text { interface }\end{array}$ & $\begin{array}{l}\text { Outdoor/ } \\
\text { Indoor }\end{array}$ & Open to all & Cheap \\
\hline Femtocell & $<23$ & $<50 \mathrm{~m}$ & $\begin{array}{l}\text { Home } \\
\text { Broadband }\end{array}$ & Indoor & $\begin{array}{l}\text { Closed } \\
\text { Subscriber } \\
\text { group }\end{array}$ & Very Cheap \\
\hline Relay & 30 & $300 \mathrm{~m}$ & Wireless & $\begin{array}{l}\text { Outdoor/ } \\
\text { Indoor }\end{array}$ & Open to all & Cheap \\
\hline RRH & 46 & Few Km & $\begin{array}{l}\text { Fiber Optic } \\
\text { Cable }\end{array}$ & Outdoor & Open to all & Cheap \\
\hline
\end{tabular}


increasing the network capacity and coverage is the inclusion of a different kind of a small cell known as Metrocell or Urbancell. Metrocells are typically layered onto the macro network and operate in licensed spectrum. Such types of network structures are a relatively new in the HCNs. Metrocell is much smaller than a Macrocell and it operates at low RF power with a coverage area of about $100 \mathrm{~m}$. Metrocells can be mounted easily inside/outside the buildings, transport hubs and other urban areas.

Another aspect in HCNs is the coverage and capacity optimization. These are the significant factors that describe the performance of the system [8], which are degraded by different types of interference. To realize the promised capacity and coverage gains with much reduced interference in HetNets, Madan et al. [9] described model for design and operation which mainly focuses on cell splitting, range expansion, semi-static resource negotiation on third-party backhaul connections, and fast dynamic interference management for QoS via over-the-air signaling. For the deployment of heterogeneous macro-pico, the authors consider (i) novel association schemes to take into account the different transmit powers of pico and macro base-stations, and (ii) joint association and resource allocation algorithms which are fair across cells and maximize system efficiency. Whereas in [10], the coverage probability of HetNets with n-interacted transmission nodes is analyzed statistically. The proposed strategy is based on the network co-operation (NC) and interference cancellation (IC) which requires the real time interaction between the transmission nodes. And in the context of EE analysis, an information theoretic approach is used in [11], where all the interference signals are taken into consideration. Here, the EE of the interference limited network is shown as a function of the BS transmission power and a gradient based optimal solution is expressed for the maximization of EE. In addition, different techniques such as cooperation among Macro-eNBs (MeNB) and Femeto-eNBs (HeNB) and collaborative frequency scheduling [12], formation of groups of HeNBs and exchange of information (path loss, geographical location, etc.) among neighboring HeNBs [13], power control [14, 15] and intelligent spectrum access [16] have been analyzed and discussed to reduce co-tier and cross-tier interference.

The organization of the rest of the paper is as follows: interference in HetNet and its various sources are given in Section 2, an overview of various interference management techniques used are presented in Section 3, a survey of different interference mitigation techniques and their challenges are given in Sections 4, and 5 concludes the paper. 


\section{Interference in Hetnet}

Practically, HetNet is a network densification approach to support ever increasing traffic where large number of transmission nodes being deployed in a small geographical area [17]. Network densification comprises of (i) spatial densification, (ii) spectral aggregation. Spatial densification is realized by increasing the number of antennas per node (user device and/or base station), and increasing the density of base stations deployed in the given geographic area, ensuring nearly uniform distribution of users among all base stations. Where, using large amount of electromagnetic spectrum stretched from $500 \mathrm{MHz}$ to millimeter wave bands $(30-300 \mathrm{GHz})$ is realized as spectral aggregation. In general, there are two approaches for spectrum allocation between the Macrocell and Femtocell users: (i) spectrum splitting and (ii) spectrum sharing. Due to the scarcity of available spectrum, the Picocell, Femtocell and Relays operate in the licensed spectrum owned by the Macrocell network [18]. Interference is caused when two cellular networks use the same frequency bands (Co-Channel Interference-CCI) or adjacent frequency bands (Adjacent Channel Interference-ACI) for communication purposes [19]. Due to the scarcity of spectrum availability, the frequency reuse $[20,21]$ technique is widely used to improve the cell capacity. Considering the co-channel interference in the downlink (DL) as one of the most biggest challenges in HetNets, in the recent LTE releases Inter-Cell Interference Coordination (ICIC) and Coordinated Multi-Point (CoMP) is introduced as an approach for interference mitigation as interference is considered to be one of the major limiting factors of the performance of HetNets. The 3GPP LTE Intercell Interference Coordination (ICIC) [22], is a technique to confront the inter-cell interference, however it requires coordination between neighboring cells, both in terms of exchanging information regarding subscribers at one cell and their interference level on other neighboring cells, as well as coordination in the resource allocation, which further complicates the scheduling process.

The use of HetNets facilitates a better spatial reuse of the spectrum which on the other hand leads to higher interference scenarios. In a frequency reuse network, the UE usually follows on the cell with the strongest received DL signal (SSDL). The point where SSDL is the same for both cells is considered as the border between two cells. In-order to get more advantages from HetNets, a technique called Cell Range Expansion (CRE), where the area served by the low power small cell can be increased, is applied to offload more data traffic 
from the Macrocell to the smallcells, which causes additional interference. In HetNets an increased interference on the DL is experienced by the UE located in the CRE region and served by the base station in the small cell. In a two-tier HetNet comprising Macrocells and Femtocells, the two types of interference occurring in the DL are shown in Figure 2.

- Co-tier (Co-layer) interference: This type of interference occurs between neighboring Femtocells.

- Cross-tier interference (Cross-layer): This type of interference occurs between Femtocells and Macrocells.

In a two-tier cellular network which uses OFDMA technique, such types of interferences (Co-tier/Cross-tier, Uplink/Downlink) occur due to the use of same band/sub-channel for communication. Again in addition to the large number of created cell boundaries, the interference problem in HetNets is especially challenging due to the following reasons [6]:

- Unplanned Deployment. The Low-power nodes such as Femtocells are moved or switched on/off at any time though they are deployed in an ad-hoc manner by users. Again due to deployment of low power cells like Femtocells, the radio frequency (RF) interference will arise from Femto-Femto, Femto-Macro, and Macro-Femto cell. So the traditional network planning and optimization schemes are not so efficient because operators are not able to control neither the number nor the location of these cells. So it is difficult to set any centralized interference avoidance or management scheme.

- Closed Subscriber group (CSG) Access. When a cell restricts its access to some users and only a few users are permitted to access, this is known

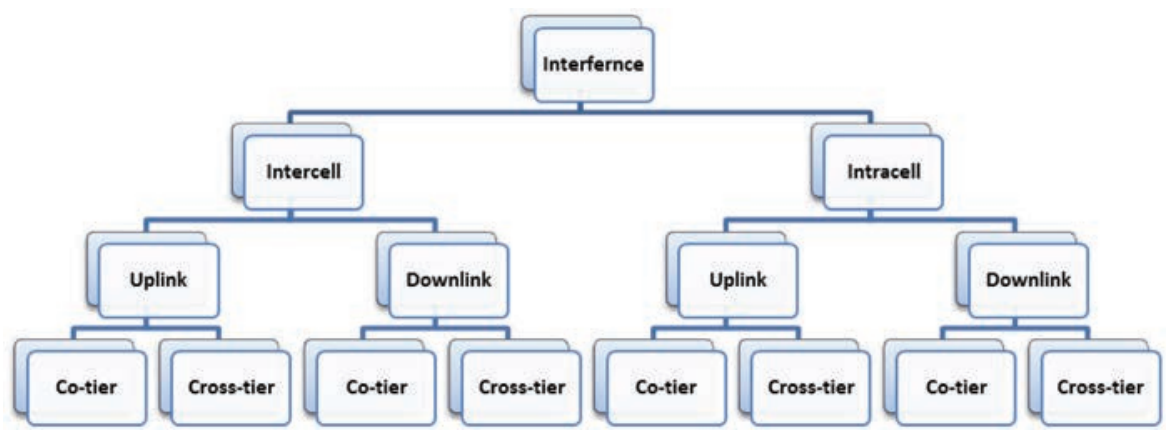

Figure 2 Classification of interference in HetNets. 
as CSG mode. In this mode of operation non-subscribers may not be allowed to be connected to the nearest BS and forced to be connected to a far serving Macrocell. Hence, a nonsubscriber transmits at high power in the uplink (UL) to compensate for the path losses thus causing the interference scenario worsen. Again during the DL the HeNB of the CSG interferes with the micro user equipments (MUEs) which are connected to the far MeNB.

- Power Difference between the Nodes. In HetNets, due to the large difference in the transmission power between Macrocells and other lowpower nodes, most of the UEs tend to connect to Macrocells, instead to the small cells at the shortest path loss distance, which causes uneven load distribution between the cells and leads to overloading Macrocells. Due to this server selection procedure in the DL, users connected to Macrocells will severely interfere with all low-power nodes located in their vicinity in the UL.

- Cell Range Expansion (CRE). Normally, UEs connect to the cell that provides the strongest DL received signal. But in the case when all the UEs are connected to the Macrocell because of their large transmit power rather than to Picocells located at a shorter distance with less number of serving UEs, the traffic load will be unevenly distributed. This will cause the Macrocell to be overloaded whereas the Picocells will be underutilized $[23,24]$. To remedy to this problem, the concept of CRE [25] was introduced as a new cell selection procedure, In this technique, the DL power of a low power BSs increases so that more users can be associated to the low power BSs. Due to CRE the offload users which will get adverse channeling effect from the low power BSs will get a strong interference from the high power BSs.

A complete scenario comprising of different interference in HCNs is shown in Figure 3, where it is assumed that the cell structure is hexagonal.

Again considering the case of Metrocell deployment, it is quite easy for the Mobile Network Operators (MNO) to manage the interference effectively for indoors. But it is too problematic if the Metrocells are mounted outdoors, because in a LTE network the Macro and Metrocells operate at the same set of frequencies. Usually, the interference is most likely to affect the UEs located at the cell edge but due to the strong transmitted power of MeNB, most of the coverage area of the Metrocell is under-the-cover as shown in Figure 4, where a 3 sectored cell is considered. 


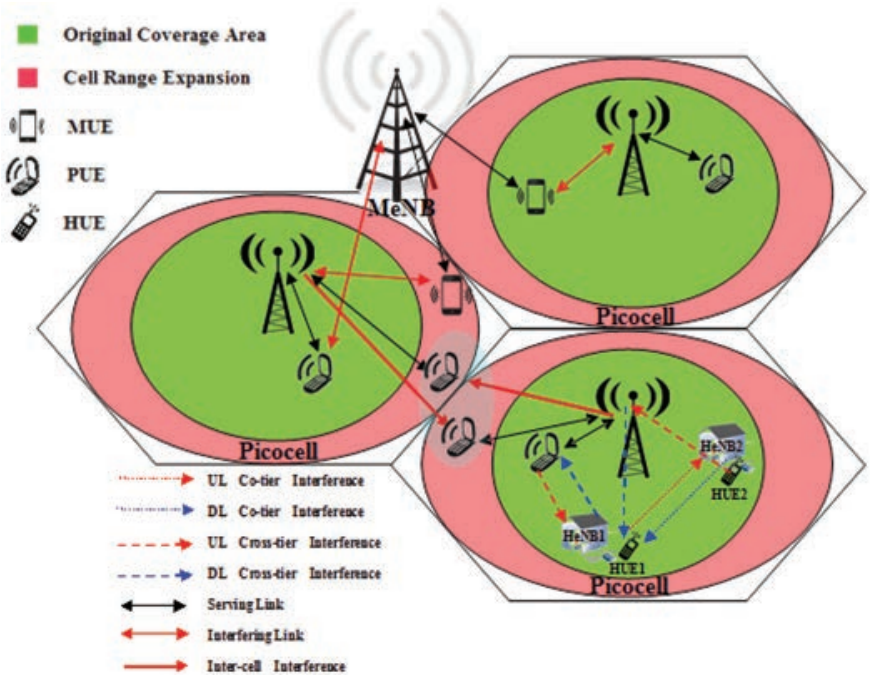

Figure 3 Different Interference Scenarios in HetNet.

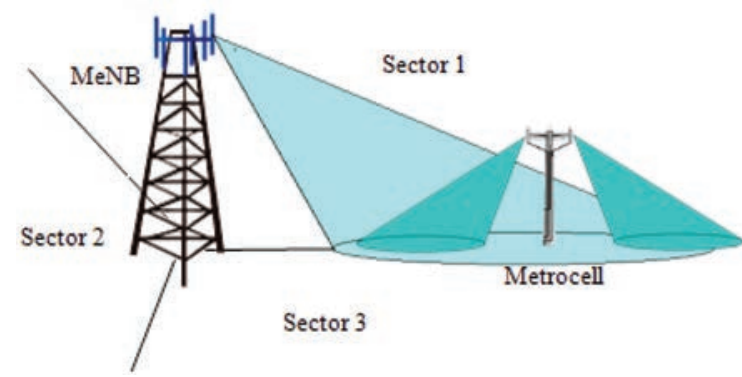

Figure 4 The Interference between Macrocell and Metrocell.

\section{Interference Management Techniques and their Challenges}

During the last decade, extensive research in the inter-cell interference (ICI) management has been carried out and a significant progress has been achieved $[26,27]$. As the next generation wireless mobile communication system follows a large change in its network structure and planning of deployment, there are so many challenges toward the direct application of conventional inter-cell interference management schemes. To cope up with the different technical challenges along with the efficient interference mitigation various techniques have proposed by researchers that vary depending on the specific 
scenarios and physical layer of technology. The major key features of the nextgeneration wireless communication systems are more data centric services instead of voice services, IP core services, dynamic services (mobility support) and heavy upload traffic. In order to cope with the changes, the next-generation ICI management should evolve [28]:

- Set the objective of providing a maximal data rate.

- Deal with the changes in interference mechanism triggered by the new physical-layer technologies.

- Take the form of joint management of power allocation and user scheduling.

- Handle the time-varying channel conditions in mobile environment.

- Operate in a fully distributed manner.

- Increase the spectral efficiency while avoiding centralized coordination of resource allocation of the users in the uplink channel.

To address these objectives many different approaches have been considered in the literature.

Mostly to achieve high performing dense wireless networks, efficient and low complexity interference mitigation techniques are required for HCNs. So while designing an efficient interference mitigation technique some other aspects e.g. environmental, installation and antenna parameters can also be taken into account for the successful deployment of HCNs. Some key challenges to mitigate the interference in multi-tier heterogeneous cellular networks, as a result of the comparison between the existing cell association and power control schemes are as follows [29]:

- Designing Optimized Cell Association and Power Control (CAPC) Methods for Multi-Tier Networks: Though the optimal solutions for downlink CAPC problems may not be optimal for the UL due to the various aspects such as, status of the BSs and state of the channel of each UE. In order to optimize this issue, joint optimization frameworks must be provided or as proposed in [7], the mobile users can connect with two different BSs for uplink and downlink transmissions, which are expected to be the case in $5 \mathrm{G}$ multi-tier cellular networks.

- Designing Efficient Methods to Support Simultaneous Association to Multiple BSs: In order to increase the throughput and to reduce the cell outage ratio the CAPC method can be further extended where an UE can able to associate with multiple BSs and to decide the condition for selecting the BSs during UL or DL. 
- Designing Efficient Methods for Cooperation and Coordination among Multiple Tiers: A UE may experience the highest DL power from the Macrocell, whereas the highest UL path gain may be from a nearby small cell. In this case the UE can associate to the Macrocell in the DL and to the small cell in the UL. In this scenario CoMP scheme can be used to mitigate the interference in the network which requires strong integration between low power nodes and the network in order to obtain the user's location and channel state.

In earlier stage of mobile communications a number of classical time and frequency domain ICI mitigation approaches e.g. Power Control, Interference Averaging, Scheduling and Multiuser Detection were used. But currently a number of features added to the 3GPP LTE and LTE-A specification that can be used to mitigate the interference problem in HetNets with small cells [30]:

- LTE: Inter-Cell Interference Coordination (ICIC).

- Carrier aggregation (CA) with cross-carrier scheduling (CS).

- LTE-A: Enhanced ICIC (eICIC) which is an adjusted version of ICIC for HetNet, and Coordinated Multi-Point (CoMP) [26, 27] which uses Channel Status Information (CSI) reported by UE.

Furthermore, to reduce the interference in HetNets, eICIC techniques have been developed for 3GPP LTE Release 10, which is divided into three major categories [31] as, Time-domain techniques [32], Frequency-domain techniques and Power control techniques. But based on the network deployment and considering different aspects of interference, the Interference Management techniques can be divided into following categories:

- Decentralized Interference Management [33]: In these techniques such as fractional frequency reuse, advanced receivers, distributed power control and static resource partition there is no coordination in between cells [34-36].

- Coordinated Interference Management: In these techniques, the neighboring cells coordinate with each other by overhead messaging in order to manage the interference in the network mutually, e.g. enhanced inter-cell interference coordination (eICIC), joint power control and coordinated multipoint (CoMP) communications.

- Resource management based Interference Management [37, 38]: The main objective of Resource Management/Radio Resource Management (RRM) in HCNs includes, resource allocation among Macrocells and smallcells, Radio Admission Control (RAC), handover management, 
packet scheduling etc. Depending upon the evolved network architecture and enhanced technologies, there are several challenges that arise in resource management like, interference mitigation, QoS, resource utilization etc. Several associated works have been proposed to focus on both uplink [39-44] and downlink [45] power control in two-tier Femtocell.

In general, RRM schemes can be classified into centralized, decentralized and hybrid approaches. But based upon the under-laying technologies and working principle, the RRM can be classified into resource allocation, frequency scheduling, femto-aware spectrum allocation, graph theory, Femtocell clustering, cognitive radio, distributed learning, frequency reuse, co-operative approach, power control approach etc.

- Hardware based Interference Management [37]: There are several interference management techniques which are hardware based like the direction antenna, a multichannel multi-antenna-based beamformingcodebook-restriction strategy, which enables the Femtocell users to select the channels that are least influenced by Cross-tier interference [46].

In particular no specific classification standard criterion for interference management techniques exists in HCNs. Most of the interference management techniques are designed in combination with resource allocation schemes. Based upon different air interface technologies, some different interference management techniques are discussed below.

\subsection{Fractional Frequency Reuse}

In HetNets due to the co-deployment of high power and low power nodes in the same network using the same spectrum, more advanced interference coordination and radio resource management schemes are required than in the traditional cellular networks in order to achieve a high network capacity and good user experience. So Fractional Frequency Reuse (FFR) [47, 48] scheme comes into focus as an ICIC technique for OFDMA based LTE network due to its low complexity. In this technique the cells are divided into two spatial regions i.e. inner region and outer region. Then a frequency reuse factor is applied in each region to reduce the ICI between the cell-zone MUE and center-zone MUE, as well as in between the neighboring cell-zone MUEs by applying an efficient channel allocation method. The FFR scheme can be deployed in two ways i.e. Strict FFR and Soft Frequency Reuse (SFR) for both uplink and downlink. SFR can provide higher cell-edge throughput in comparison with the Strict FFR by increasing the power control factor but 
this comes at the cost of decreased inner region throughput [48]. This scheme is not efficiently applicable to dynamic cellular networks due to the random distribution of traffic and network structure in the spatio-temporal coordinate. In order to cope with such type of dynamic cellular networks, Imran et al. [49] have proposed a Self Organizing framework for adaptive Frequency Reuse and Deployment (SO-FRD) which can be implemented independently in each region without any explicit signaling and coordination between the regions. This scheme is highly scalable with low operational complexity and can be applicable in both short and long time scale. An improved FFR scheme known as, Optimal Static Fractional Frequency Reuse (OSFFR) shows that the average network sum rate is better in comparison with other schemes as well as this scheme provides higher SINR with much reduced co-tier and crosstier interference [50]. Although the OSFFR scheme increases the number of useable sub-channels per unit area but in a scenario of mass deployment of $\mathrm{HeNB}$, it is too difficult to provide the desired data rate along with the efficient target blocking capabilities.

\subsection{Power Control Techniques}

Power Control techniques are one of the most important aspects in-order to mitigate the interference issues in HetNets. The most important parameters to compare the performance of different power control schemes are, (i) Aggregate transmit power, (ii) Outage ratio, (iii) Aggregate throughput. So the objective of power control approach, is to support a user with its minimum acceptable throughput and a system with maximum aggregate throughput, but the main problem with this technique is that the reduction of the radiated power at a Femtocell also leads to reduction of the total throughput of Femtocell users, which may significantly improve the performance of victim Macro Users (MUEs). Summarizing of different power control techniques in HCNs, a hierarchical structure is shown in Figure 5.

\subsubsection{Different power control techniques for interference mitigation in HetNets}

The different power control approaches can be divided into three major categories [31], as Proactive or reactive power control, Cluster-based power control, Game-theoretic power control. Descriptively, the proactive or reactive power control schemes mainly focus on reducing the transmission power of HeNBs and dynamic or adjustable power setting for fixed HeNB power setting. It is operated in two ways (i) Open-loop power control method where the HeNB 


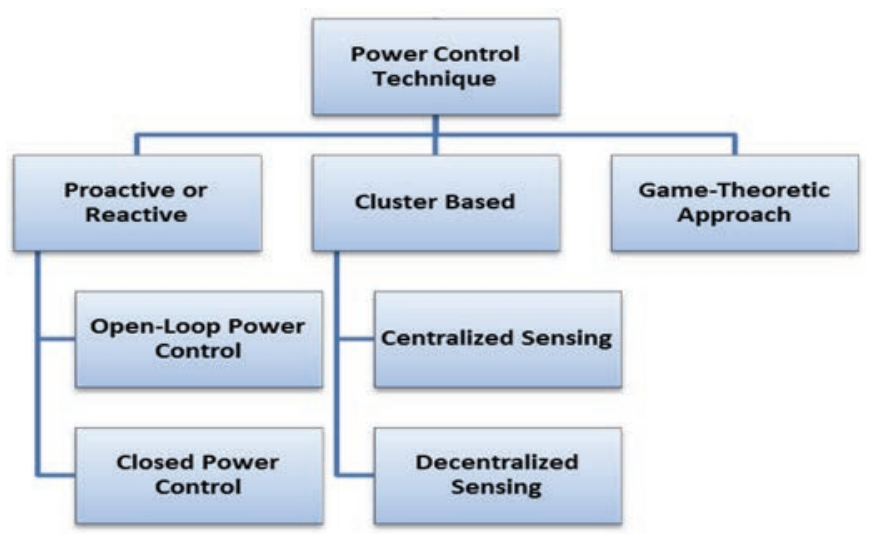

Figure 5

adjusts its transmission power based on its measurement results in a proactive manner and (ii) Closed-loop power control method where the HeNB adjusts its transmission power based on the coordination with MeNB in a reactive manner. The Cluster-based power control approach proposes a centralized sensing scheme to obtain information about how many is the number of active Femtocells per cluster by an MeNB and broadcast the interference allowance information to Femtocells for their initial power setting, while distributed sensing can be used to sense the number of active Femtocells per cluster by an HeNB and to adjust its initial power setting correspondingly. In Gametheoretic power control approach, the distributed power control techniques are designed and analyzed for Femtocells, where the MeNBs and HeNBs compete with each other in-order to maximize their capacity under power constraint situations [31]. This approach is followed due to the uncertainty of the SINR values in the wireless cellular network and in most of the cases the output is the Nash Equilibrium of the power control game. The different power control techniques in a Femtocell network are shown in Figure 6.

Adaptive network sensing (ANS) technique for DL power control is proposed in [51] to reduce the ICI caused by uncoordinated deployment of Femtocell in HetNets, bringing up throughput performance enhancement. Overall energy efficiency could also be achieved with proper power control and power consumption optimization. A distributed power control and resource utilization algorithm to minimize the transmit power and intracell interference is proposed in [52]. Different approaches for power optimization are also considered for the uplink in many research works. Taking into 


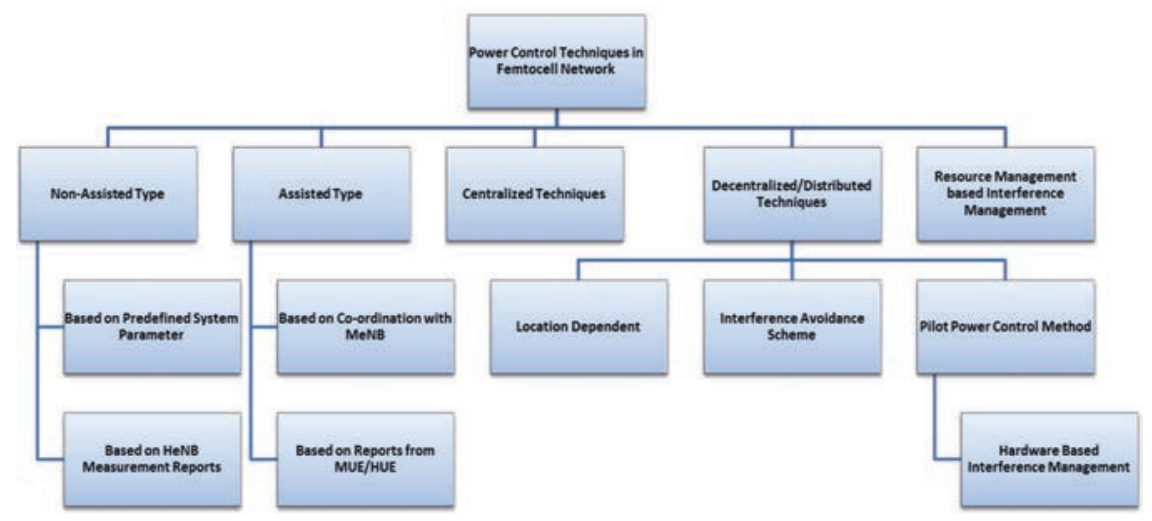

Figure 6 Different Power Control Techniques for Interference Mitigation in HetNets.

account the uncertainty of channel state information (CSI) and depending upon the different service requirements in two-tier Femtocell network systems, a robust hierarchical uplink power control scheme has been proposed by [53]. The approach is based on a game theoretic approach applying a distributed iterative power control algorithm to obtain Nash Equilibrium with the goal of maximizing the utilities of all users. The authors in [54] propose a power control scheme for two-tier small cell networks formulated as a Stackelberg game focused on the reduction of the cross-tier and co-tier interference together with as the power consumption of the users. Other uplink power control schemes based on combined open-loop and closed loop approaches and heuristics are proposed in [55-57].

\subsection{Cognitive-based Approach}

The cluster-based power control approach above proposes a centralized sensing scheme to obtain information about the number of active Femtocells per cluster by the help of a MeNB and to broadcast the interference allowance information to Femtocells for their initial power setting. In Cognitive-based approaches, the network utilizes the distributed sensing scheme to sense the number of active Femtocells per cluster by an HeNB and adjusts its initial power setting correspondingly. Based on the information collected by applying cognitive-based approach, the Gibbs sampler, game-theoretic and compressive sensing approaches have been explored more for interference mitigation (both cross-tier and co-tier) in a Macro-Femto HetNet [58]. The cognitive-based interference mitigation technique is basically used to control 
the cross-tier and co-tier interference in OFDMA based Femtocell HetNets. The interference management IM based on the cognitive approach can be classified into three categories as, opportunistic interference avoidance, and interference alignment and interference cancellation.

In order to collect the information about the communication environment the HeNB is enabled with cognitive radio (CR) technology by which different challenges like random deployment of Femto-Macro BS, backward compatibility, lack of coordination between MeNB and HeNB and restricted access can be avoided. In [59] an overview of the different possible CR enabled interference mitigation techniques e.g. Cognitive Resource Block Management, Cognitive Interference Cancellation for Retransmission, Strategic Game-Based Resource Block Management, Gibbs-Sampler-Based Spatial Path Selection, have been considered to control cross-tier and cotier interference in OFDMA Femtocell heterogeneous networks. In order to mitigate the interference, both in co-tier and cross-tier, Li Huang et al. [60] propose GSOIA(Gale-Shapley Opportunistic Interference Avoidance) scheme for Macro-Femto HetNets. In this scheme the cognitive enabled Femtocells randomly senses the communication environment to find free spectrum which reduces the cross-tier interference and the Gale-Shapely spectrum sharing provides one-to-one matching policy (by executing this policy among nearby Femtocells) causing a reduction in co-tier interference.

\subsection{Q-learning Approach}

The ICIC technique plays a vital role in the CRE mechanism. Due to CRE the cell-edge users suffer from low DL SINR, as the ICIC manages to provide an improved SINR to the extended range users in the DL. In time-domain ICIC technique the MeNB reduces its transmission power by using Almost Blank Sub-frame (ABS), where in frequency-domain ICIC technique the CA \& CS is used to avoid the co-tier interference in the DL. For both, time and frequency-domain ICIC, Simsek et al. [61] propose a dynamic reinforcement learning based on eICIC for the HetNets comprising of Macrocell and Picocell. Here the Picocell learn their CRE bias and DL power transmission and the Macrocell transmission power can be optimized by applying a decentralized Q-learning procedure (inspired form the Reinforcement Learning (RL)) for joint interference management and cell association mechanism. The Macrocell optimizes its transmission by serving its own users while adhering to the Picocell interference constraint. By comparing the simulation results of the proposed solution with a number of existing ICIC approaches, such as resource 
partitioning (RP), fixed cell range expansion (CRE) and fixed Almost Blank Subframe (ABS) significant gains of up to $125 \%$ are seen as compared to RP and $23 \%$ as compared to static ICIC approaches.

\subsection{Coordinated Approach}

The authors in $[62,63]$ propose a framework based on the iterative waterfilling approach to be applicable in the mobile environment depending upon its adequate speed. To realize this ICI management scheme coordination among the cells is necessary. Depending upon the quality of the back-haul link the coordination between the cells can be implemented in two ways i.e. semi-static and dynamic coordination approach. Dynamic coordination provides better performance due to its flexibility as compared to the semi-static coordination. But the area of coverage of the dynamic coordination is smaller than that of semi-static coordination. In dynamic coordination joint transmission and coordinated scheduling or beamforming can be used to reduce ICI. To achieve the benefits of both the coordination in an efficient way a Hybrid coordination scheme is used, which gives a significant gain of more than $28 \%$ average throughput gain and more than $30 \%$ cell edge throughput gain over semi-static coordination with different bias values [64].

\subsection{Resource Allocation Approach}

Focusing on co-tier interference, Kaimaletu et al. [65] propose a cognitive resource allocation interference management scheme on the DL, which is able to deal with co-tier and cross-tier interference in heterogeneous cellular wireless networks comprising of Macrocells and Femtocells. Here depending upon the ability to cause interference with each others UEs, the Macrocells and Femtocells get the transmission opportunities and allocated resources (in time and frequency which are near/fully orthogonal), assuming that the Femtocells are operated in closed subscriber group. Considering no interference management scenario, when this scheme is compared with frequency reuse techniques this scheme significantly enhances the average cell-edge UE throughput (5\%-tile throughput) with slight degradation in overall sum throughput. It is observed that without any interference management, there is up to $270 \%$ increase in cell-edge throughput for the MUEs and 50\% when the reuse factor is 3, whereas for HUEs the increase is about $140 \%$. Also the scheme decreases the probability of cell-edge users in the system experiencing degraded SINR. In [66] the authors propose a cognitive-based interference management solution by sharing measured pathloss information 
among neighbors and selecting component carriers (CC), according to the estimated mutual interference. Based on the existing distributed methods $[67,68]$, this approach sets an improved cognitive based spectrum selection scheme by which each Femtocell is able to select spectrum intelligently by considering the interference from neighboring Femtocells, as well as the potential interference caused to the neighbor cell by itself, so that a balance between overall capacity and frequency reuse efficiency can be achieved.

\subsection{Beamforming}

Among the different approaches that have been studied for interference management since the last decade, the hardware based approach attracts more researchers to focus on it in recent years. In this approach integration of different arrangements of the antenna pattern is done which mainly focuses on the shape of the beam of the serving cell in order to reduce the interference caused to the other cell. By selecting a proper antenna pattern and phase angle the coverage and capacity of the serving cell can be optimized, hence the throughput can be increased.

A commonly used hardware based technique is known as beamforming. In this technique the serving cell focuses the response of the beam in a particular direction, while reducing its response in all other direction. This causes a reduction in interference to the other cells. A spatial filter is used to process the data obtained from an array of sensors to enhance the quality of the received signal based on the surrounding noise and interference. The objective is to estimate the signal arriving from desired direction in the presence of noise and interfering signals.

The beamforming operation can be performed in two ways [69], (i) Conventional beamforming and (ii) Adaptive Beamforming. The conventional beamforming can be done in either frequency-domain or in timedomain. The delay in time-domain corresponds to linear phase shift in frequency domain, where the adaptive beamforming optimizes a collection of weight vectors used to locate targets in a noise environment. A beamforming which samples the propagating wave field in space and the output at time ' $\mathrm{k}$ ' can be represented as shown below [70],

$$
Y(k)=\sum_{i=1}^{J} w_{i}^{*} X_{i}(k)
$$

Where, $\mathbf{J}=$ Number of sensors

$$
\mathrm{w}^{*}=\text { Conjugate weight }
$$


Another beamforming which samples the propagating wave field in both space and time can be represented as [70],

$$
Y(k)=\sum_{i=1}^{J} \sum_{m=0}^{k-1} w_{i . m}^{*} X_{i}(k-m)
$$

The Equations (1) \& (2) can be written as [57],

$$
Y(k)=w^{H} X(k)
$$

Where, $\mathrm{w}^{\mathrm{H}}=$ weight vector

$\mathrm{X}(\mathrm{k})=$ Data vector

$\mathrm{H}=$ Hermition transpose/Complex conjugate

As interference mitigation is an important aspect in a two-tier (MacroFemto) network, since the HeNBs are generally connected to the MeNBs the backhaul signalling is limited for the interference co-ordination in Femtocell network. To reduce the signalling overhead and computational complexity, a distributed beamforming technique is proposed in [71] to mitigate the interference in the downlink as well as to improve the throughput of a multiple input single output (MISO) Femtocell network. In this method an optimal structure of the beamforming vector is presented initially and then based on the desired expression and the Karush-Kuhn-Tucker (KKT) condition, a closed-form solution of the distributed beamforming is proposed. In CSG mode handover problems arise due to the lack of co-ordination between adjacent cells. The authors in [72] propose a method for transmit beamforming based interference control to mitigate the downlink CCI from the uncoordinated adjacent HeNBs. This method utilizes multiple antennas in the HeNBs in conjunction with a control-only connection established between UE and interfering HeNBs. Here the work has been carried out by considering an extension of the twoantenna transmit beamforming configuration which is used in High Speed Downlink Packet Access (HSDPA )framework, to a four-antenna that can be used in LTE standards. A joint Femtocell clustering and selective beamforming scheme is proposed in [73] to mitigate the interference at HeNB during the uplink transmission of MUE in HetNets. In this proposed clustering scheme the coverage of the HetNet is splited and then the dominating interference sources are confined in each cluster. This causes the reduction in interference at the HeNB by eliminating the interference in clusters. Then to eliminate the in-cluster interference a MinGI (Minimize the Generated Interference) based selective beamforming is proposed. 
In Co-ordinated multi-point (COMP), the co-ordinated beamforming is more efficient in comparison with the joint transmission (JT). Though the JT improves the spectral efficiency of the network it increases the complexity and is highly expensive to be practically implemented. Based on the coordinated beamforming technique, the author [74] proposes a heuristic beamforming algorithm for the interference mitigation in a two-tier HetNets. By this approach the load distribution can be improved by utilizing the average channel gain for the selection of BS and the spectral efficiency can be increased by considering a set of interfered users of the selected BS. The proposed approach in [75] is based on the design of an orthogonal beam forming technique to mitigate the cross-tier interference in Femtocell networks. The strategy of the beam forming is achieved by selecting a subset of the antenna. It is selected based on the number of MUEs, the intensity of the HeNBs and SNR of the users. The idea is to improve the system throughput through the tradeoff between the gain in multiplexing and multiuser interference (cross-tier). This mode of beam selection significantly reduces cross-tier interferences and ensures radio resources to the HeNBs in an opportunistic fashion. The scheme further minimizes cross-tier interference through the deployment of a distributed power control mechanism for HeNBs.

\subsection{Antenna Tilt Approach (Directional Antenna)}

Another hardware based interference management technique is the antenna tilting approach. To meet the different QoS and performance requirements in terms of SINR, several researches have been done to show the impact of optimization in the antenna parameters for the future wireless communication systems [76, 77]. In contrast, the concept of self-organizing networks (SON) is being introduced by $3 \mathrm{GPP}$ to make the network more autonomous and time efficient for future generation cellular networks, where a part of SON use cases defines the coverage and capacity optimization (CCO). Hence, considering the coverage and capacity as a key factor of HetNets, antenna tilting technique attracts more researchers to focus on it due to its low operational expenditure (OpEX) and complexity. By using antenna tilting technique, a significant improvement in the desired SINR, average throughput and sum data rate within the cell can be observed as well as reduction in the interference to the other cells can also be achieved $[78,79]$. Furthermore, the coverage and capacity of the network can also be improved in the constraints of available bandwidth. To optimize the capacity and coverage, the antenna tilting (Uptilt/Downtilt) is possible in two ways i.e. mechanically or electrically (as shown in Figure 7) 

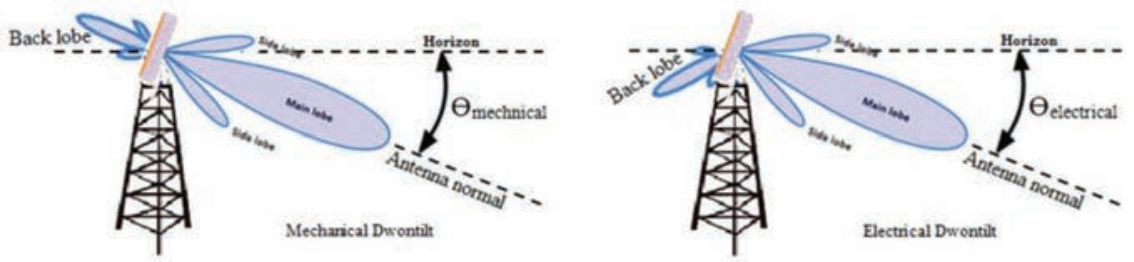

Figure 7 Antenna tilting scheme.

depending upon the number of users and their distribution in a current network environment.

The mechanical tilt is cost inefficient and more time consuming though it requires physical site visits. But by using remote electrical tilt (RET) the radiation pattern can be adjusted remotely. By utilizing the electrical downtilt, the HeNB signal transmission can be controlled at the cell's edge while well-designed upper side-lobe suppression (USLS) sharpens the cell's edge to minimize interference. By optimizing the angle of antenna tilt depending upon the antenna height and distance between them the desired SINR values can be achieved for communication purposes. The objective of the antenna tilting scheme is to confine the HeNB transmitting power and simultaneous reuse of radio resources in multiple cells. By confining the transmitting power to each cell (sectrorized cell) using directional-beam antennas the co-channel interference can be reduced, thus increasing radio resource efficiency [80]. The optimization using antenna tilt is more useful in the case when a hotspot of users is formed in between different cells. The nearest HeNB adjusts its antenna tilt towards the hotspot zone, thus reducing the interference from the other cells. This causes a significant improvement in the SINR level. The adaptation of antenna tilt is formulated as an optimization task for utility fairness in the cellular network, subject to network constraints [81]. The antenna tilting adjustments can be carried out jointly at the HeNB in coordinated manner for interference management. This optimization problem is normally non-convex, but the authors in [81] show that: (1) Under high SINR operating mode and with an appropriate choice of variables, the optimization is convex for any concave utility function, and (2) under any SINR operating mode, the optimization can be formulated in a convex manner when the objective is a proportional fair rate allocation. This approach is much simple as making use of measurements which are readily available at the base station, but this optimization is only applicable for certain conditions. 


\section{S. R. Samal}

A significant improvement in performance due to CCO by using RET can be observed in case of suboptimal network planning or reuse of $3 \mathrm{G}$ network planning [82]. In this article, the authors propose a case based learning (CBL) centralized algorithm which mainly relies on the cases and instances (e.g. received power, SINR, call drops) that are stored in the memory for applying them to the new situations directly. Due to the low implementation complexity, high accuracy in case of small number of instances and irrelevant features the CBL is considered to be more accurate. According to this algorithm, instances/samples are collected from the central network server and stored in the memory. Then the optimum antenna tilt is determined using the $\mathrm{k}$-nearest neighbor algorithm where the case matches to the current state. The main drawback of this algorithm is that, it is not clear about the number of cases/instances to be collected.

In [83], a method is proposed for self-coverage and capacity optimization using antenna tilt in a fully distributed manner. The CCO in this method uses fuzzy reinforcement learning (RL) approach namely Q-learning, which operates in fully autonomous fashion without any priori information for the network conditions. The simulation results show convergence of the solution to the global optimal settings and $20 \%$ improvement in performance in comparison to other existing fuzzy logic based RL approach. But this method lacks to provide a significant throughput gain. A decentralized fuzzy Q-learning is proposed in [84] to optimize the base station antenna downtilt automatically using information form its own and neighboring cells. This scheme uses a central control mechanism for initialization and termination of the optimization processes. The simulation results show significant improvement in performance gain in terms of coverage and capacity and fast convergence. The advantage of this algorithm is that, it has the capability to learn from the changing environment and may also provide other self-optimizing capabilities for self-organizing LTE network.

To avoid the problems of algorithm in $[82,83]$ i.e. incapability of handling a large set of network configurations and the inability to adapt to the network environment without prior knowledge, a centralized RL sparse sampling algorithm is proposed in [85]. The authors of [85] focus on the problem of self-optimization in the LTE network environment by adjusting antenna tilt and show that this approach is more efficient than supervised learning and Q-learning algorithms in terms of self-healing performance and multiple coverage problems. The authors in [86] add dynamic and adaptive antenna tilt adjustment for the best trade-off between coverage and capacity in mobile networks. This approach is based on RL methodology with low computational 
complexity for distributed real-time operation. Based on the simulation results, it gives nearly $30 \%$ improvements in the sum data rate that can be achieved. The advantages of the proposed algorithm is that it is model independent and can be applicable to the current and future mobile networks for ICIC, load balancing and energy saving.

Finally, in a HetNet comprising of Metrocells, the area of spectral efficiency and average user rate can be increased by the application of optimal electrical downtilt in order to decrease the metro cell antenna vertical beamwidth [87]. For the successful deployment of Metrocells in HetNets the operators can apply Macrocell principles to the metro cell network design to minimize metro cell interference and optimize spectral efficiency. In [88], it is demonstrated that, by utilizing the electrical downtilt and USLS the improved spectral utilization enables the operators to increase user throughput or satisfy more users with savings in capital expenditure (CapEx)/OpEx.

\section{Advanced Interference Management}

Basically, most of the interference management operations in 4G LTE system are carried out in the network side only, because of their high backward compatibility and easy deployment. But the sole management of interference issues in the network side arise lots of overhead and practical complication issues [89, 90]. To avoid this problem LTE Release 12 focuses on UE-side interference management. Still more things about this technique are to be unveiled in this release. Based on the key challenges of $4 \mathrm{G}$ and implementation of UE-side interference management, the Advanced Interference Management (AIM) technique may be the source of attraction for the future $5 \mathrm{G}$ wireless networks [91]. The main advantage of this technique is that, it is suitable for both UE-side as well as the network side operation. In [91] the authors discuss some important issues related to the interference and requirements for AIM in 5G systems dealing with two aspects of AIM:

- UE-side interference management by advanced receivers with interference joint detection/decoding;

- Network-side interference management by joint scheduling.

In UE-side interference management, the receiver (commonly referred as advanced receiver) reconstructs the interference signal by considering the values of the coding scheme, channel and resource allocation as well as the output of detector/decoder. In the network-side, the authors apply a joint scheduling approach for interference mitigation which is very much similar 
to CoMP scheme. The authors also address a number of practical issues that lead towards real implementation, such as realistic interference conditions, practical receiver architectures, CSI reporting for AIM, practical issues with joint scheduling, prospective gains etc. The benefit of these technologies is the improvement in the cell edge throughput without immolating the cell average throughput in comparison with the current LTE system performance, which may be considered as key factor for $5 \mathrm{G}$.

Towards the deployment of a bigger number of small cells in HCNs, a Self-Interference Cancellation (SIC) technique is proposed in [92], which can be used to reduce the backhaul problems by re-using radio resources for transmission and reception purpose simultaneously. SIC plays an important role for simplifying the spectrum management issues. Furthermore in the constraints of spectrum availability, the huge demand of data consumption, low latency, high throughput can be met by utilizing the SIC technique in enhanced interference coordination, spectrum virtualization and increased link capacity in a cost-effective way.

\section{Conclusion and Future Research}

Due to the proliferation of wireless cellular networks the demand for high data rates also increases. It is too difficult for the MNOs to meet all the requirements in an efficient way while maintaining the desired SINR level. So for future cellular communication systems, more focus is given on the deployment of a large number of small cells operating on higher frequencies. In this context HCNs attract more attention, where small cells like Picocell, Femtocell, Metrocell are overlaid to the conventional Macrocell network. For the successful deployment of heterogeneous wireless cellular networks, it is desired to consider the interference as key challenge. There are different aspects of interference which arise due to heterogeneity nature of the HCNs and random spatiotemporal distribution of the mobile users. So it is necessary to mitigate the interference in various ways to provide a better QoS in a cost and time efficient way as well as to increase data rates, capacity and efficiency of the network.

In this paper different types of interference management techniques of HCNs were considered. The main focus was related to the hardware control based and power control based interference management techniques. Analyzing the existing approaches and considering the problems due to the extensive growth in deployment of Femtocell like intra-interference (FemtoFemto), dense-deployments and environmental impact (global warming and 
greenhouse gas emissions) the development of a framework for power control in dense Femtocell deployments, with a main focus on the energy efficiency of cellular communication networks could be a way forward to the development green communication solution. In this relation it will be necessary to optimize base station transmission power, to manage/reduce the interference and to maximize the energy efficiency in LTE/LTE-A systems. Along with this, dynamic trade-off between coverage and network complexity as well as tradeoff between coverage and capacity in mobile networks must be implemented to boost the efficiency of HCNs. In addition, the power control approach and electrical beam tilting could be conjugated to provide optimized coverage depending upon the user density, mobility or distribution and also to avoid the network coverage holes.

Nevertheless all the work done in this field, a lot of future research should be still focused on the topics related to resource management and allocation, as these issues will play a major role in terms of either spectrum or power efficient utilization. Orthogonal resource allocation should also be considered as a major approach for reduction of the cross-tier interference, but more efficient methods should be investigated and developed, as this approach requires more frequency bands for spectrum allocation. With the limited available spectrum efficient interference management techniques with low computational complexity will be necessary to be developed. Looking towards the future $5 \mathrm{G}$ wireless communications, research and the running standardization efforts by ITU, IEEE, 3GPP, etc, it is obvious that $5 \mathrm{G}$ will lead to ultra-dense deployment of small cells and IoT nodes, paving the way to the introduction of cell-less architectures and new communication scenarios especially in smart cities [93]. The idea behind cell-less architectures will bring up the needs to find new flexible user association schemes, cooperative communication approaches and other novel access methods to cope with the complex interference scenarios in a smart city [94]. This will impose new research challenges related to power control and interference. Research on energy efficiency in $5 \mathrm{G}$ through the convergence of computation and transmission power together is also another open challenge for future research [95].

\section{References}

[1] Poulkov, V., (2016). Beyond the next generation access in R. Prasad, S. Dixit (eds) Wireless world in 2050 and beyond: A window into the future!, Springer Series in Wireless Technology. ch. 2, 17-39. 
[2] Cisco. (2012). Cisco visual networking index: Global mobile data traffic forecast update, White Paper.

[3] Ericsson. (2011). Differentiated mobile broadband, White Paper.

[4] Metis. (2013). Scenarios, requirements and KPIs for 5G mobile and wireless system, ICT-317669 METIS project.

[5] Kyoseva, T., et al. (2014). Disruptive innovations as a driving force for the change of wireless telecommunication infrastructures, Springer Journal, Wireless Personal Communications, 78(3), 1683-1697, doi: 10.1007/s11277-014-1911-z

[6] Lopez-Perez, D., et al. (2011). Enhanced intercell interference coordination challenges in heterogeneous networks, in IEEE Wireless Communications, 18(3), 22-30, doi: 10.1109/MWC.2011.5876497

[7] Bendlin, R. et al. (2011). From homogeneous to heterogeneous networks, A 3GPP Long Term Evolution Rel. 8/9 Case Study, in 45 Annual Conference on Information Sciences and Systems (CISS), pp. 1-5, Baltimore, MD.

[8] Hegde, N. and Altman, E. (2003). Capacity of multiservice WCDMA networks with variable GoS, IEEE Wireless Communications and Networking (WCNC-2003), pp. 1402-1407, vol. 2, New Orleans, LA, USA, Mar. 2003.

[9] Madan, R., et al. (2010). Cell association and interference coordination in heterogeneous LTE-A cellular networks, IEEE Journal On Selected Areas In Communications, 28(9), 1479-1489.

[10] Samal, S. R., et al. (2017). Coverage analysis of heterogeneous wireless network with n-interacted transmission nodes, IGI Global, International Journal of Interdisciplinary Telecommunications and Networking (IJITN), 9(4), 49-58, doi: 10.4018/IJITN.2017100106.

[11] Bandopadhaya, S., et al. (2017). Base station transmission power optimization in interference-limited cellular networks for maximum energy efficiency, in Proceedings of $13^{\text {th }}$ International Conference on Advanced Technologies, Systems and Services in Telecommunications (TELSIKS), pp. 228-231, Nish, Serbia.

[12] Sahin, M. E., Guvenc, I., and Jeong, M. (2009). Handling CCI and ICI in OFDMA femtocell networks through frequency scheduling, in IEEE Transactions on Consumer Electronics, 55(4), 1936-44.

[13] Li, H., Xu, X., and Hu, D. (2010). Graph method based clustering strategy for femtocell interference management and spectrum efficiency improvement, in $6^{\text {th }}$ International Conference on Wireless Communications Networking and Mobile Computing (WiCOM), pp.1-5, Chengdu. 
[14] Park, S., et al. (2010). Beam subset selection strategy for interference reduction in two-tier femtocell networks, IEEE Transactions on Wireless Communications, 9(11), 3440-49.

[15] 3GPP R1-106052. (2010). Per cluster based opportunistic power control, 3GPP RAN1 Meeting, Jacksonville, FL.

[16] Zhang, L., Yang, L., and Yang, T. (2010). Cognitive interference management for LTE-A femtocells with distributed carrier selection, in $72^{\text {nd }}$ IEEE Vehicular Technology Conference Fall (VTC 2010-Fall), pp. 1-5.

[17] Bhushan, N., et al. (2014). Network densification: the dominant theme for wireless evolution into 5G, in IEEE Communications Magazine, 52(2), 82-89.

[18] Kang, X., Zhang, R., and Motani, M. (2012). Price-based resource allocation for spectrum-sharing femtocell networks: A Stackelberg game approach, in IEEE Journal on Selected Areas in Communications, 30(3), 538-549.

[19] Mishra, A. R. (2007). Advanced Cellular Network Planning and Optimization 2G/2.5G/3G...Evolution to $4 G$, John Wiley \& Sons Ltd, pp. 71-77.

[20] Rao, G. S. (2012). Mobile Cellular Communication, $1^{\text {st }}$ Ed., PEARSON International, pp. 235-260.

[21] Rappaport, T. S. (2007). Wireless Communications: Principles and Practice, $2^{\text {nd }}$ Ed., Prentice Hall PTR, pp. 105-155.

[22] Boudreau, G., et al. (2009). Interference coordination and cancellation for 4G networks, IEEE Communication Magazine, 47(4), 74-81.

[23] Lopez-Perez, D., Chu, X., and Guvenc, I. (2012). On the expanded region of picocells in heterogeneous networks, in IEEE Journal of Selected Topics in Signal Processing, 6(3), 281-294.

[24] Guvenc, I., et al. (2011). Range expansion and inter-cell interference coordination (ICIC) for picocell networks, IEEE Vehicular Technology Conference (VTC Fall), pp. 1-6, San Francisco.

[25] Guvenc, I. (2011). Capacity and fairness analysis of heterogeneous networks with range expansion and interference coordination, IEEE Communication Letters, 15(10), 1084-87.

[26] Lee, B. G., Park, D., and Seo, H. (2008). Wireless Communications Resource Management, John Wiley \& Sons, Singapore.

[27] Katzela, I., and Naghshineh, M. (1996). Channel assignment schemes for cellular mobile telecommunication systems: A comprehensive survey, in IEEE Personal Communications, 3(3), 10-31. 
[28] Kwon, H., et al. (2008). Inter-cell interference management for nextgeneration wireless communication systems, in Journal of Communications and Networks, 10(3), 258-267.

[29] Hossain, E., et al. (2014). Evolution toward 5G multi-tier cellular wireless networks: An interference management perspective, in IEEE Wireless Communications, 21(3), 118-127.

[30] Do, M., and Son, J. (2014). Interference coordination in LTE/LTE-A (1): inter-cell interference coordination (ICIC), tech@netmanias.com.

[31] Hossain, E., Le, L. B., and Niyato, D. (2014). Radio Resource Management in Multi-Tier Cellular Wireless Networks, Wiley Publication.

[32] Zhang, D., et al. (2013). The time-domain enhanced inter-cell interference coordination in heterogeneous networks, in $19^{\text {th }}$ European Wireless Conference (EW-2103), pp. 1-5, Guildford, UK.

[33] Chandrasekhar, V. and Andrews, J. G. (2009). Spectrum allocation in tiered cellular networks, in IEEE Transaction Communications, 57(10), 3059-3068.

[34] Chandrasekhar, V., et al. (2009). Power control in two-tier femtocell networks. IEEE Transactions on Wireless Communications 8(8), 43164328.

[35] Damnjanovic, A., et al. (2011). A survey on 3GPP heterogeneous networks, in IEEE Wireless Communications, 18(3), 10-21.

[36] Khandekar, A., et al. (2010). LTE Advanced: heterogeneous networks, in European Wireless Conference (EW-2010), pp. 978-982, Lucca.

[37] Chai, X., et al. (2015). A user-pairing power control algorithm in two-tier HetNet, in $81^{\text {st }}$ IEEE Vehicular Technology Conference (VTC Spring), pp. 1-5.

[38] Lee, Y. L., et al. (2014). Recent Advances in Radio Resource Management for Heterogeneous LTE/LTE-A Networks, in IEEE Communication Surveys \& Tutorials, 16(4), 2142-2180.

[39] Wang, Y. and Venkatraman, S. (2012). Uplink power control in LTE heterogeneous networks, in $4^{\text {th }}$ IEEE International Workshop on Heterogeneous and Small Cell Networks (HetNets), Globecom Workshops (GC Wkshps), pp. 592-597.

[40] Sun, Y., Jover, R. P., Wang, X. (2012). Uplink interference mitigation for OFDMA femtocell networks, in IEEE Transactions on Wireless Communications, 11(2), 614-625.

[41] Li, J. (2013). Uplink power control for heterogeneous networks, IEEE Wireless Communications and Networking Conference (WCNC), pp. 773-777. 
[42] Poulkov, V., et al. (2014). Combined power and inter-cell interference control for LTE based on role game approach, Springer Journal, Telecommunication Systems, 55(4), 481-489, doi: 10.1007/s11235-013-9803-1

[43] Koleva, P., et al. (2015). Risk assessment based LTE hetnet uplink power and interference control, in Proceeding of the $38^{\text {th }}$ International Conference of Telecommunications and Signal Processing, pp. 210-214, Prague, Czech Republic, July 2015.

[44] Koleva, P., et al. (2012). Interference limited uplink power control based on a cognitive approach, in Proceedings of International Conference on Telecommunications and Signal Processing (TSP), pp. 242-246, Prague, Czech Republic.

[45] Sun, D., et al. (2011). Downlink power control in cognitive femtocell networks, IEEE Wireless Communication and Networking Conference, pp. 1-5, Nanjing.

[46] Park, S., et al. (2011). A beamforming codebook restriction for crosstier interference coordination in two-tier femtocell networks, IEEE Transactions Vehicular Technology, 60(4), 1651-1663.

[47] Novlan, T. D., et al. (2011). Analytical evaluation of fractional frequency reuse for OFDMA cellular networks, in IEEE Transactions on Wireless Communications, 10(12), 4294-4305.

[48] Elfadil, H. E. E. O. M., Ali, M. A. I., and Abas, M. (2015). Fractional frequency reuse in LTE networks, in $2^{\text {nd }}$ World Symposium on Web Applications and Networking (WSWAN), pp. 1-6, Sousse.

[49] Imran, A., Imran, M. A., and Tafazolli, R. (2010). A novel self organizing framework for adaptive frequency reuse and deployment in future cellular networks, in $21^{\text {st }}$ Annual IEEE International Symposium on Personal, Indoor and Mobile Radio Communications, pp. 2354-2359, Instanbul.

[50] Saquib, N., Hossain, E., and Kim, D. I. (2013). Fractional frequency reuse for interference management in LTE-advanced hetnets, in IEEE Wireless Communications, 20(2), 113-122.

[51] Hassan, T. U., et al. (2018). Interference management in femtocells by the adaptive network sensing power control technique, Future Internet, 10(3), 25, doi: 10.3390/fi10030025

[52] Yang, Z., et al. (2016). Power control and resource allocation for multi-cell OFDM networks, in IEEE Conference on Computer Communications Workshops (INFOCOM WKSHPS), pp. 891-896. San Francisco, CA.

[53] Liu, Z., et al. (2017). Robust power allocation based on hierarchical game with consideration of different user requirements in two-tier femtocell networks, Elsevier Computer Networks, 122, 179-190. 
[54] Jiang, Y., et al. (2018). Power control via Stackelberg game for small-cell networks, arXiv: 1802.04775 [cs.IT].

[55] Gochev, H., Poulkov, V., and Iliev, G. (2013). Improving cell edge throughput for LTE using combined uplink power control, Springer Journal, Telecommunication Systems, 52, 1541-1547, ISSN: 1018-4864.

[56] Asenov, O., Koleva, P., and Poulkov, V. (2013). Heuristic approach to dynamic uplink power control in LTE, in Proceedings of International Conference on Telecommunications and Signal Processing (TSP), pp. 235-238, Rome, Italy.

[57] Koleva, P., et al. (2014). Improved open loop power control for LTE uplink, in Proceedings of International Conference on Telecommunications and Signal Processing (TSP), pp. 183-187, Berlin, Germany.

[58] Cheng, S. M., and Chen, K. C. (2013). Cognitive radios to mitigate interference in macro/femto heterogeneous networks, Heterogeneous Cellular Networks, pp. 119-144, doi: 10.1002/9781118555262.ch6

[59] Cheng, S. M., et al. (2011). On exploiting cognitive radio to mitigate interference in macro/femto heterogeneous networks, in IEEE Wireless Communications, 18(3), 40-47.

[60] Huang, L., Zhu, G., and Du, X. (2013). Cognitive femtocell networks: an opportunistic spectrum access for future indoor wireless coverage, in IEEE Wireless Communications, 20(2), 44-51.

[61] Simsek, M., Bennis, M., and Czylwik, A. (2012). Dynamic inter-cell interference coordination in hetnets: A reinforcement learning approach, in Wireless Networking Symposium, Globecom-2012, Anaheim.

[62] Han, Z., Ji, Z., and Liu, K. J. R. (2007). Non-cooperative resource competition game by virtual referee in multi-cell OFDMA networks, in IEEE Journal on Selected Areas in Communications, 25(6), 1079-1090.

[63] Kwon, H. and Lee, B. G. (2006). Distributed resource allocation through non-cooperative game approach in multi-cell OFDMA systems, in IEEE International Conference on Communications, pp. 4345-4350, Istanbul.

[64] Gao, Q., et al. (2012). Interference management in heterogeneous network, in $7^{\text {th }}$ International Conference on Communications and Networking in China, 379-383, Kun Ming.

[65] Kaimaletu, S., et al. (2011). Cognitive interference management in heterogeneous femto-macro cell networks, in IEEE International Conference on Communications (ICC), pp. 1-6, Kyoto.

[66] Zhang, L., Yang, L., Yang, T. (). Cognitive interference management for LTE-A femtocells with distributed carrier selection, in $72^{\text {nd }}$ IEEE Vehicular Technology Conference Fall (VTC 2010-Fall), pp. 1-5, Ottawa. 
[67] Claussen, H. (2006). Distributed algorithms for robust self-deployment and load balancing in autonomous wireless access networks, in IEEE International Conference on Communications, pp. 1927-1932, Istanbul.

[68] Garcia, L. G. U., Pedersen, K. I., and Mogensen, P. E. (2009). Autonomous component carrier selection: interference management in local area environments for LTE-advanced, IEEE Communications Magazine, 47(9), pp. 110-116.

[69] C. R. Prasanth, et al. (2013). Beam forming and adaptive beam forming techniques and its implementation on ADSP TS 201 processor, IOSR Journal of VLSI and Signal Processing (IOSR-JVSP), 3(5), pp. 07-17.

[70] Van Veen, B. D., and Buckley, K. M. (1988). Beamforming: A versatile approach to spatial filtering, in IEEE ASSP Magazine, 5(2), 4-24.

[71] Bak, J., et al. (2013). Interference mitigation techniques for femtocell networks, International Symposium on Intelligent Signal Processing and Communication Systems, pp. 251-256, Naha.

[72] Husso, M., et al. (2010). Interference mitigation by practical transmit beamforming methods in closed femtocells, EURASIP Journal on Wireless Communications and Networking, Article ID 186815, Springer International Publishing, doi.org/10.1155/2010/186815

[73] Zhang, D., et al. (2015). Joint femtocell clustering and selective beamforming for interference mitigation in heterogeneous networks, IEEE/CIC International Conference on Communications in China (ICCC), pp. 2-4.

[74] Oguejiofor, O., and Zhang, L. (2016). Heuristic coordinated beamforming for heterogeneous cellular network, in $83^{\text {rd }}$ IEEE Vehicular Technology Conference (VTC Spring), pp. 1-5, Nanjing.

[75] Park, S., et al. (2010). Beam subset selection strategy for interference reduction in two-tier femtocell networks, IEEE Transactions on Wireless Communications, 9(11), 3440-3449.

[76] Yilmaz, O. N. C., Hamalainen, S., and Hamalainen, J. (2009). System level analysis of vertical sectorization for 3GPP LTE, in $6^{\text {th }}$ International Symposium on Wireless Communication Systems, pp. 453-457, Tuscany.

[77] Siomina, I., Varbrand, P., and Yuan, D. (2006). Automated optimization of service coverage and base station antenna configuration in UMTS networks, in IEEE Wireless Communications, 13(6), 16-25.

[78] Athley, F., Johansson, M. N. (2010). Impact of electrical and mechanical antenna tilt on LTE downlink system performance, in $71^{\text {st }}$ IEEE Vehicular Technology Conference (VTC 2010-Spring), pp. 1-5, Taipei. 
[79] Parikh, J., and Basu, A. (2014). Impact of base station antenna height and antenna tilt on performance of LTE systems, IOSR Journal of Electrical and Electronics Engineering (IOSR-JEEE), 9(4), 06-11.

[80] Cho, H. S., Kim, Y. I., and Sung, D. K. (2002). Protection against cochannel interference from neighboring cells using down-tilting of antenna beams, in $53^{\text {rd }}$ IEEE Vehicular Technology Conference, vol. 3, pp. 1553-1557, Rhodes.

[81] Partov, B., Leith, D. J., and Razavi, R. (2015). Utility fair optimization of antenna tilt angles in LTE networks, IEEE/ACM Transactions on Networking, 23(1), 175-185.

[82] Yilmaz, O. N. C., Hamalainen, J., and Hamalainen, S. (2010). Selfoptimization of remote electrical tilt, in $21^{\text {st }}$ IEEE International Symposium on Personal Indoor and Mobile Radio Communications (PIMRC), pp. 1128-1132, Instanbul.

[83] Razavi, R., Klein, S., and Claussen, H. (2010). Self-optimization of capacity and coverage in LTE networks using a fuzzy reinforcement learning approach, in $21^{\text {st }}$ IEEE International Symposium on Personal Indoor and Mobile Radio Communications (PIMRC), pp. 1865-1870, Instanbul.

[84] Li, J. et al. (2012). Self-optimization of coverage and capacity in LTE networks based on central control and decentralized fuzzy Qlearning, International Journal of Distributed Sensor Networks, 8(8), doi: org/10.1155/2012/878595

[85] Thampi, A., et al. (2012). A sparse sampling algorithm for selfoptimisation of coverage in LTE networks, International Symposium on Wireless Communication Systems (ISWCS), pp. 909-913.

[86] Dandanov, N., et al. (2017). Dynamic self-optimization of the antenna tilt for best trade-off between coverage and capacity in mobile networks, Wireless Personal Communications: Springer Link, 92(1), 251-278.

[87] Li, X., et al. (2015). Metrocell Antennas: The positive impact of a narrow vertical beamwidth and electrical downtilt, IEEE Vehicular Technology Magazine, 10(3), 51-53.

[88] Kemp, S. (2014). Improving metro cell performance with electrical downtilt and upper sidelobe suppression, Commscope White Paper.

[89] Bandemer, B., Gamal, A. E., and Kim, Y. H. (2012). Simultaneous nonunique decoding is rate-optimal, in Proceedings of the $50^{\text {th }}$ Allerton Conference, pp. 9-16, USA. 
[90] Baccelli, F., Gamal, A. E., Tse, D. N. C. (2011). Interference networks with point-to-point codes, IEEE Transactions on Information Theory, 57(5), 2582-2596.

[91] Nam, W., et al. (2014). Advanced interference management for 5G cellular networks, in IEEE Communications Magazine, 52(5), 52-60.

[92] Hong, S., et al. (2014). Applications of self-interference cancellation in 5G and beyond, in IEEE Communications Magazine, 52(2), 114-121.

[93] Han, T., et al. (2017). 5G converged cell-less communications in smart cities, in IEEE Communications Magazine, 55(3), 44-50.

[94] Ge, X., et al. (2017). Energy Efficiency challenges of 5G small cell networks, in IEEE Communications Magazine, 55(5), 184-191.

[95] Poulkov, V. (2017). The unified wireless smart access for smart cities in the context of a cyber physical system, in Proceedings of Global Wireless Summit (GWS), pp. 17-21, Cape Town, South Africa.

\section{Biography}

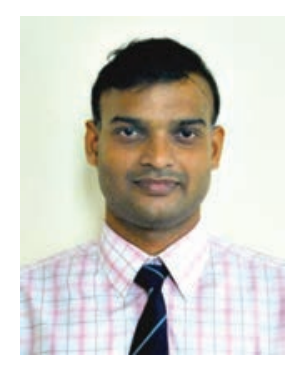

Soumya Ranjan Samal is a Ph.D. student at the Technical University of Sofia at Sofia, Bulgaria since 2016. He received his B.E. in Electronics \& Instrumentation Engineering from Biju Patnaik University of Technology, Bhubaneswar, India in 2004. Soumya then went on to pursue his M.E. in Computer Science \& Engineering from the Utkal University of Bhubaneswar, India in 2009. He, as an Assistant Professor in Silicon Institute of Technology, Bhubaneswar, India has acquired a solid experience about 12 years of teaching in Communication Engineering. Soumya also worked as a Project Engineer in Indian Institute of Technology, Bombay, India in 2005. His Ph.D. work centers on Interference Management in cellular network and discusses the green communication movement to develop energy efficient solutions through antenna parameters. 
